\title{
Characteristics of TiVN and TiVCN Coatings by Cathodic Arc Deposition
}

\author{
Wei-Yu Ho ${ }^{1, a}$, Ming-Der Chen ${ }^{1}$, Chien-Liang Lin ${ }^{1, b}$, Woei-Yun $\mathrm{Ho}^{2, \mathrm{c}}$ \\ ${ }^{1}$ Department of Materials and Energy, MingDao University, Taiwan \\ ${ }^{2}$ Department of Applied Physics, National Pingtung University, Taiwan

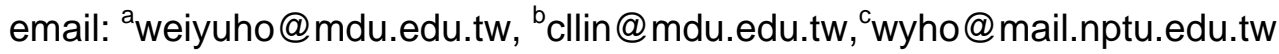

Keywords: Cathodic arc deposition, Hard coating, Wear, Thermal stability

\begin{abstract}
Currently, TiVN and TiVCN coating are not prevailing in the literature. Only a few publications are revealed with basic properties. In this study, the TiVN and TiVCN coatings are deposited by using cathodic arc deposition system with $\mathrm{Ti}$ and $\mathrm{TiV}$ alloy dual targets. The result shows that $\mathrm{V}$ and $\mathrm{C}$ addition lead to the increase of surface roughness by droplets deposition in the TiVN and TiVCN coatings as compared to TiN. However, grain size of the TiVN and TiVCN coatings is obviously reduced by adding the $\mathrm{V}$ and $\mathrm{C}$ in the coating. The highest hardness and the best wear resistance are obtained for the TiVCN coating. Thermal stability of the TiVCN coatings is affected by the $\mathrm{C}$ addition resulting in severe oxidation.
\end{abstract}

\section{Introduction}

Titanium vanadium nitride (TiVN) has not been widely published in the literatures yet. It is expected that TiVN possesses better performance of their hardness and tribological behaviors with respect to TiN coating. TiVCN as well as TiVN hard coatings can be the other potential wear resistance coatings which make it can replace the conventional TiN coatings. During decades, ternary transition metal nitrides such as $(\mathrm{Ti}, \mathrm{Al}) \mathrm{N}$ and $(\mathrm{Cr}, \mathrm{Al}) \mathrm{N}$ have been attracting great interest for applications as hard protective coating materials due to their high hardness, good wear resistance and excellent corrosion protection [1-6]. Extensively investigations have extended to multilayered TiAlN/CrAlN [7-8] and additional metal dope [9-10] ternary transition metal nitrides. However, among those researches, only a few studies of $(\mathrm{Ti}, \mathrm{V}) \mathrm{N}$ have been reported in the literature [11-17]. Knotek O. et al. [11] reported that arc-evaporated Ti-V-N coatings exhibited high hardness and excellent thermal stability. The case of TiVN-coated inserts showed excellent performance in turning operations. Tao et al. [15] reposted V implantation by the MEVVA ion source implantation technique to improve the mechanical and tribological properties of TiN coatings. The tribotest showed the V-implanted TiN coatings had a lower friction coefficient as well as a better wear resistance. Fernandes et al. [16, 17] revealed the effect of $\mathrm{V}$ additions on the structure, mechanical properties and oxidation resistance of $\mathrm{Ti}-\mathrm{Si}-\mathrm{V}-\mathrm{N}$ coatings deposited by $\mathrm{DC}$ reactive magnetron sputtering. It is expected that TiVCN as well as TiVN coatings may be available in a diverse range of applications in industry, based on their hardness and tribological behavior. Therefore, the aim of this research is to explore the properties of the TiVN and TiVCN coatings deposited by cathodic arc deposition technique (CAD).

\section{Experimental procedure}

TiN, TiVN and TiVCN coatings were finished by a CAD machine equipped with two circular (dia. $10.2 \mathrm{~mm}$ ) cathodes installed face to face. A high purity TiV (99.9\%) metal, and a high purity Ti (99.9\%) metal were used as targets. Polished tungsten carbide and stainless steel substrates were used for analysis. The substrates were mounted in a substrate holder giving a target to substrate distance of $150 \mathrm{~mm}$. A stoichiometric TiN coating was deposited from the Ti target to serve as reference. In order to enhance the adhesion of the TiVN and TiVCN coatings, Ti and TiN adhesion layers were deposited on the substrates before coatings. The top layer of TiN+TiVN and TiCN+TiVCN multilayered coatings were finished, respectively. The depositions were performed 
with a negative substrate bias of $150 \mathrm{~V}$.

The surface morphologies and fractured cross-section morphology and the thickness of the coatings were investigated by scanning electron microscopy (SEM). The chemical composition of the coatings was evaluated by X-ray photoelectron spectroscopy (XPS). Crystallographic structure was investigated by $\mathrm{X}$-ray diffraction ( $\mathrm{X}$ ' PertPro MPD diffractometer) using a grazing incidence angle of $2{ }^{\circ}$ and $\mathrm{Cu} \mathrm{K} \alpha$ radiation $\left(\lambda=1.54060^{\circ} \mathrm{A}\right)$. The grain size of the coatings is calculated based on the Scherrer's formula [18]. Further, profilometer was used to evaluate surface roughness. The hardness of the coatings was measured by a Vicker's hardness equipment with the applied load of 25g. The wear test was carried out by using ball-on-disc tester with the applied load of $5 \mathrm{~N}$. The friction coefficient against tungsten carbide ball was measured in ambient condition. The dynamic oxidation resistance of the coatings was evaluated by thermogravimetric analysis (TGA/DSC) in air heated with a constant temperature ramp of $20^{\circ} \mathrm{C} / \mathrm{min}$ from room temperature up to $1000^{\circ} \mathrm{C}$. The weight gain of the samples was evaluated using a microbalance with an accuracy of $0.01 \mathrm{mg}$.

\section{Results and Discussion}

Fig. 1 shows the surface morphologies of the TiN, TiVN and TiVCN coatings by CAD. Compared to TiN coating, the higher amount of micro-droplets that resulted in increase of the surface roughness on the TiVN and TiVCN coatings was observed. The effect of the $\mathrm{V}$ and $\mathrm{C}$ content leads to increase of micro-droplets spread on the coating surface. Recently, results showed the residual stress field of the coating can be modified by the presence of surface micro-droplets, whose main effect is to increase in magnitude of the surface compressive stress and to reduce the in-depth stress gradient [19]. Therefore, higher amount of droplets on the TiVN and TiVCN coatings may affect the surface roughness but not affect the hardness and grain size which are shown in the following results.

Fig. 2 shows the cross-section microstructure of the TiN, TiVN and TiVCN coatings. All the coatings were dense structure. Actually, TiVN and TiVCN coatings are designed with multilayered structure such as TiN+TiVN and TiCN+TiVCN, respectively. The thicknesses of the films as determined from the images in Fig. 2 are given in Table 1. It was observed that the thickness of the coatings is in the range from $667 \mathrm{~nm}$ of TiN, $1083 \mathrm{~nm}$ of TiN+TiVN and 987nm of TiCN+TiVCN coatings, respectively. The elemental chemical composition of the coatings determined by XPS is shown in Table 1. The nitrogen content of the TiN coatings was close to 54 at.\% which shows a little nitrogen rich. TiVN coating contains $\mathrm{Ti}$ element of $43 \mathrm{at} \%$ and $\mathrm{V}$ of $4.7 \mathrm{at} . \%$, while the nitrogen remain the similar percentage. Because the $\mathrm{V}$ atom ions are smaller in size than the Ti ions, the lattice constant decreases when the amount of $\mathrm{V} /(\mathrm{V}+\mathrm{Ti})$ increases [12]. The TiVN coating with higher hardness than TiN meaning the solid solution strength is the dominant mechanism. Significant high amount of $\mathrm{C}$ content were detected in the TiVCN coating leading to the highest hardness among the coatings, resulting from the incorporation of nitride and carbide in the coating.

Table 1 Characteristics of the TiN, TiVN and TiVCN coatings

\begin{tabular}{|l|l|l|l|l|l|l|l|}
\hline Coating & $\begin{array}{c}\mathrm{Ti} \\
(\mathrm{at} . \%)\end{array}$ & $\begin{array}{c}\mathrm{V} \\
\text { (at.\%) }\end{array}$ & $\begin{array}{c}\mathrm{N} \\
(\mathrm{at} . \%)\end{array}$ & $\begin{array}{c}\mathrm{C} \\
(\mathrm{at.} \%)\end{array}$ & $\begin{array}{l}\text { Thickness } \\
(\mathrm{nm})\end{array}$ & $\begin{array}{l}\text { Grain size } \\
(\mathrm{nm})\end{array}$ & $\begin{array}{l}\text { Microhardness } \\
\text { (Hv25g) }\end{array}$ \\
\hline TiN & 46.6 & $\mathrm{X}$ & 53.4 & $\mathrm{X}$ & 667 & 11.6 & 2500 \\
\hline TiN+TiVN & 43.0 & 4.7 & 52.3 & $\mathrm{X}$ & 1083 & 9.9 & 3100 \\
\hline TiCN+TiVCN & 20.7 & 9.2 & 9.5 & 60.6 & 987 & 6.6 & 4100 \\
\hline
\end{tabular}



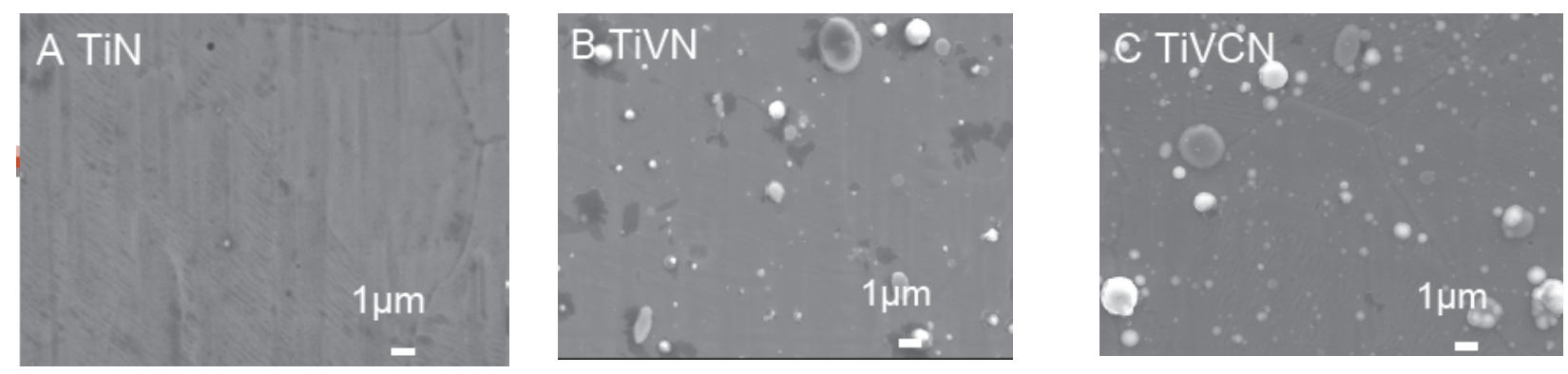

Fig. 1 Surface morphologies of the TiN, TiVN and TiVCN coatings.
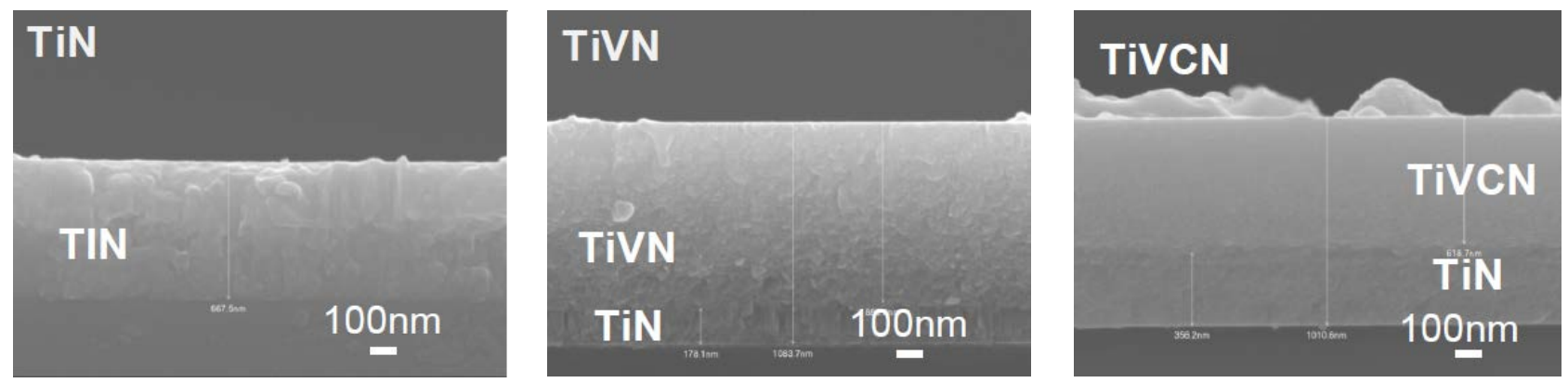

Fig. 2 Cross-section of the TiN, TiVN and TiVCN coatings.

The corresponding X-ray diffraction patterns of the as-deposited coatings are displayed in Fig. 3. TiN pattern as a reference, $\mathrm{V}$ in TiVN coating affects the diffraction peak of (200) shifted to the lower angle, as representative of lattice distortion by doping small size vanadium. In the case of TiVCN coating, the diffraction peak of (200) is further shifted to the lower angle as the C content existed. In all cases, the diffraction peaks could be generally assigned to the face center cubic (FCC) NaCl-type crystalline structure. On the other hand, residual compressive stresses of TiVN and TiVCN may evolve to higher level based on (200) peak shifted to lower diffraction angles. Based on Scherrer' equation [18], grain sizes of TiN, TiVN and TiVCN coatings were calculated as 11.6, 9.9 and $6.6 \mathrm{~nm}$, respectively. This result is related to the broadening of the (111) and (200) peaks as observed from Fig. 3. Fig. 4 shows the microhardness of the TiN, TiVN and TiVCN coatings measured by Vicker's hardness equipment under the load of 25g. Reference hardness of the TiN coating of Hv2500 is similar with the other researches. With increase of $\mathrm{V}$ and $\mathrm{C}$ contents, microhardness of TiVN and TiVCN coatings were marked with Hv3100 and Hv4100, respectively. By doping the $\mathrm{V}$ element inducing lattice distortion and decreasing grain size increase the hardness of the coatings. Results show that higher amount in the TiVCN coating forming nitride and carbide co-existed also increase the hardness of the coating.

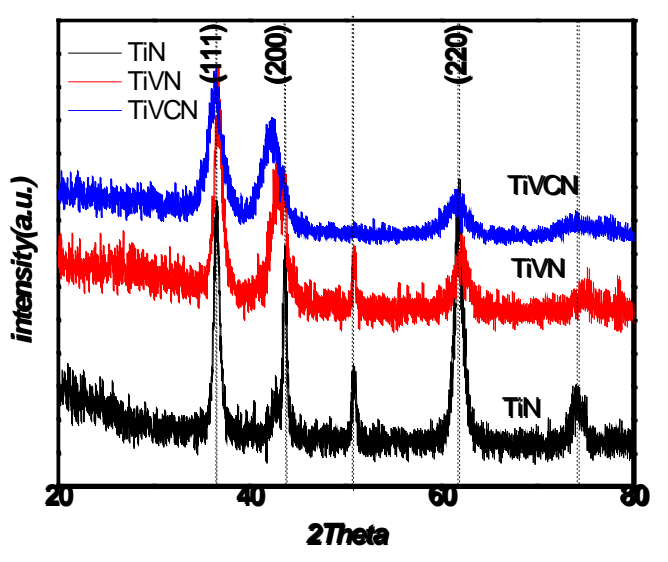

Fig. 3 XRD diffraction of the TiN, TiVN and TiVCN coatings

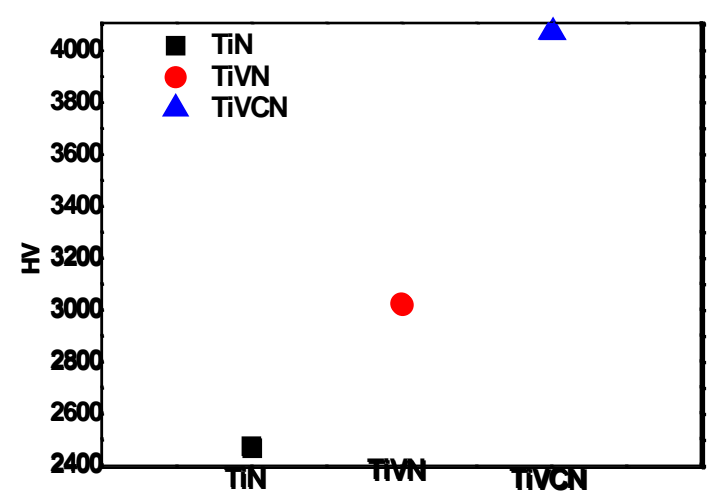

Fig. 4 Microhardness of the TiN, TiVN and TiVCN coatings measured by Vicker's hardness tester under the load of $25 \mathrm{~g}$. 
The friction coefficients of the TiN, TiVN and TiVCN under dry wear conditions are shown in Fig. 5. For TiVCN, the lowest friction coefficient is observed during wear test and the value is minimal to 0.4 . For the TiVN coating with a V content of $4.7 \mathrm{at} \%$, the friction coefficients increase quickly to a stationary value of 0.7 . The highest friction coefficient is occurred for the TiN coating. The significant difference of the friction coefficients among the coatings could be related to the difference of hardness.

Fig. 6 presents the TGA curves for TiN, TiVN and TiVCN coatings heated from room temperature to $1000{ }^{\circ} \mathrm{C}$ with a heating rate of $20 \mathrm{~K} \mathrm{~min}^{-1}$ in the flowing air. The TGA curve suggests that with increasing $\mathrm{V}$ content the mass-gain still proceeds with the similar feature with the TiN coating during the heating process. The observation of DSC curve (not shown) showing the oxidation intake temperature of TiN, TiVN and TiVCN coatings are 670, 630 and $530^{\circ} \mathrm{C}$, respectively. Small amount of $\mathrm{V}$ content in TiVN coating has no obvious protection during the oxidation process but significant increase of hardness. For TiVCN coating, after heating to the end, the distinct mass-gain of $\sim 0.2 \%$ was seen. The increasing mass-gain for the TiVCN suggests that the oxidation process strongly correlates with the less $\mathrm{Ti}, \mathrm{V}$ content and more $\mathrm{C}$ content.

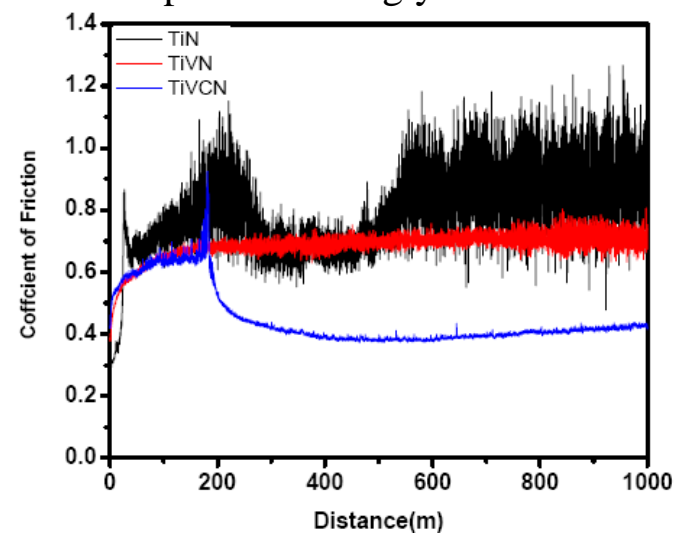

Fig. 5 The friction coefficients of the TiN, TiVN and TiVCN without lubrication conditions

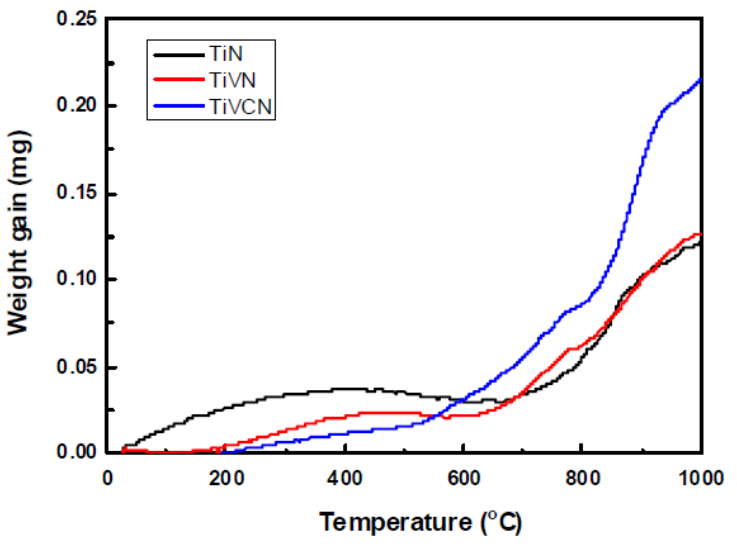

Fig. 6 the TGA curves for TiN, TiVN and TiVCN coatings heated from room temperature to $1000{ }^{\circ} \mathrm{C}$.

\section{Conclusion}

In this study, the TiVN and TiVCN coatings are deposited by using cathodic arc deposition system with Ti and TiV alloy dual targets and reactive $\mathrm{N}_{2}$ and $\mathrm{C}_{2} \mathrm{H}_{2}$ gases. The result shows that $\mathrm{V}$ and $\mathrm{C}$ addition lead to the droplets increase in the TiVN and TiVCN coatings. The grain size of the TiVN and TiVCN coatings is obviously reduced by adding the $\mathrm{V}$ and $\mathrm{C}$ in the coating. The highest hardness and the best wear resistance are obtained for the TiVCN coating. However, oxidation resistance of TiVCN is lower than that of TiN and TiVN coatings. Higher $\mathrm{C}$ element and lower metal content of TiVCN coatings is the major reason leading to oxidation process more obvious than the others.

\section{Acknowledgement}

Funding for this work was provided by the Ministry of Science and Technology, Taiwan, ROC under contract no. MOST 104-2221-E-451 -002.

\section{References}

[1]. Wenji Zhao, Fanghua Mei, Yunshan Dong, Geyang $\mathrm{Li}, \mathrm{Al}_{x} \mathrm{Ti}_{1-x} \mathrm{~N}$ hard coatings synthesized by reactive sputtering using mosaic target, Journal of Materials Processing Technology 176 (2006) 179.

[2]. G.S. Fox-Rabinovich, J.L. Endrino, B.D. Beake, A.I. Kovalev, S.C. Veldhuis, L. Ning, F. Fontaine, A. Gray, Impact of annealing on microstructure, properties and cutting performance of an 
AlTiN coating, Surface \& Coatings Technology 201 (2006) 3524.

[3]. V.H. Derflinger, A. Schütze, M. Ante, Mechanical and structural properties of various alloyed TiAlN-based hard coatings, Surface \& Coatings Technology 200 (2006) 4693.

[4]. S. Ulrich, H. Holleck, J. Ye, H. Leiste, R. Loos, M. Stuber, P. Pesch, S. Sattel, Influence of low energy ion implantation on mechanical properties of magnetron sputtered metastable ( $\mathrm{Cr}, \mathrm{Al}) \mathrm{N}$ thin films, Thin Solid Films 437 (2003) 164.

[5]. N. Bagcivan, K. Bobzin, G. Grundmeier, M. Wiesing, O. Ozcan, C. Kunze, R.H. Brugnara, Influence of HPPMS pulse length and inert gas mixture on the properties of (Cr,Al)N coatings, Thin Solid Films 549 (2013) 192.

[6]. Ying Long, Junjie Zeng, Donghai Yu, Shanghua Wu, Microstructure of TiAlN and CrAlN coatings and cutting performance of coated silicon nitride inserts in cast iron turning, Ceramics International 40 (2014) 9889.

[7]. Ping Li, Li Chen, She Q. Wang, Bing Yang, Yong Du, Jia Li, Ming J. Wu, Microstructure, mechanical and thermal properties of TiAlN/CrAlN multilayer coatings, Int. Journal of Refractory Metals and Hard Materials 40 (2013) 51.

[8]. Yuexiu Qiu, Sam Zhang, Jyh-Wei Lee, Bo Li, Yuxi Wang, Dongliang Zhao, Self-lubricating CrAlN/VN multilayer coatings at room temperature, Applied Surface Science 279 (2013) 189.

[9]. S. Mato, G. Alcala, M. Brizuela, R. Escobar Galindo, F.J. Perez, J.C. Sanchez-Lopez, Long-term high temperature oxidation of $\mathrm{CrAl}(\mathrm{Y}) \mathrm{N}$ coatings in steam atmosphere, Corrosion Science 80 (2014) 453.

[10].J.C. Sánchez-López, A. Contreras, S. Domínguez-Meister, A. García-Luis, M. Brizuela, Tribological behaviour at high temperature of hard CrAlN coatings doped with Y or Zr, Thin Solid Films 550 (2014) 413.

[11].O. Knotek, W. Burgmer, C. Stoessel, Arc-evaporated Ti-V-N thin films, Surface and Coatings Technology, 54-55 (1992) 249.

[12].N. Ichimiya, Y. Onishi, Y. Tanaka, Properties and cutting performance of (Ti,V)N coatings prepared by cathodic arc ion plating, Surface and Coatings Technology, 200 (2005) 1377.

[13].B.A. Latella, B.K. Gan, K.E. Davies, D.R. McKenzie, D.G. McCulloch, Titanium nitride/vanadium nitride alloy coatings: mechanical properties and adhesion characteristics, 200 (2006) 3605.

[14].T. Deeleard, A. Buranawong, A. Choeysuppaket, N. Witit-anun, S. Chaiyakun, P. Limsuwan, Structure and Composition of TiVN Thin Films Deposited by Reactive DC Magnetron Co-sputtering, Procedia Engineering 32 (2012) 1000.

[15].Bin Deng, Ye Tao, Deliang Guo, Effects of vanadium ion implantation on microstructure, mechanical and tribological properties of TiN coatings, Applied Surface Science 258 (2012) 9080.

[16].F. Fernandes, A. Loureiro, T. Polcar, A. Cavaleiro, The effect of increasing V content on the structure, mechanical properties and oxidation resistance of Ti-Si-V-N films deposited by DC reactive magnetron sputtering, Applied surface science, 289 (2014)114.

[17].F. Fernandes, T. Polcar, A. Cavaleiro, Tribological properties of self-lubricating TiSiVN coatings at room temperature, Surface \& Coatings Technology 267 (2015) 8.

[18].P. Scherrer, N.G.W. Gottingen, Math.-Pys. Kl. 2 (1918) 96.

[19].M. Sebastiani, M. Piccoli, E. Bemporad, Effect of micro-droplets on the local residual stress field in CAE-PVD thin coatings, Surface \& Coatings Technology 215 (2013) 407. 